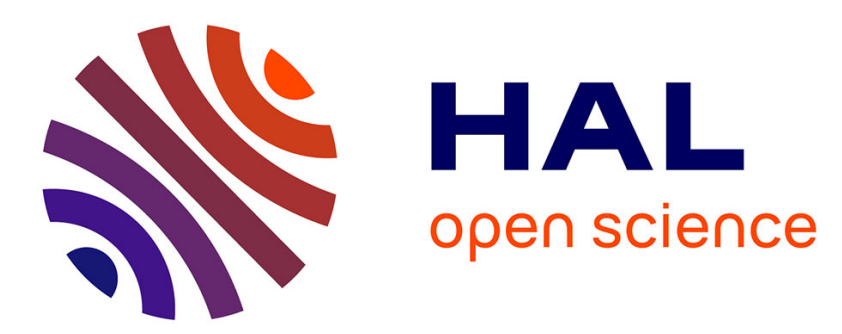

\title{
Investigation of Shock-Wave Characteristics of Porous Multi-Component Materials Under Explosive Loading by Undisturbing Electromagnetic Method
}

I. Yakovlev, V. Pai, G. Kuz'Min

\section{- To cite this version:}

I. Yakovlev, V. Pai, G. Kuz'Min. Investigation of Shock-Wave Characteristics of Porous MultiComponent Materials Under Explosive Loading by Undisturbing Electromagnetic Method. Journal de Physique IV Proceedings, 1997, 07 (C3), pp.C3-323-C3-328. 10.1051/jp4:1997357 . jpa-00255514

HAL Id: jpa-00255514

https://hal.science/jpa-00255514

Submitted on 1 Jan 1997

HAL is a multi-disciplinary open access archive for the deposit and dissemination of scientific research documents, whether they are published or not. The documents may come from teaching and research institutions in France or abroad, or from public or private research centers.
L'archive ouverte pluridisciplinaire HAL, est destinée au dépôt et à la diffusion de documents scientifiques de niveau recherche, publiés ou non, émanant des établissements d'enseignement et de recherche français ou étrangers, des laboratoires publics ou privés. 


\title{
Investigation of Shock-Wave Characteristics of Porous Multi-Component Materials Under Explosive Loading by Undisturbing Electromagnetic Method
}

\author{
I.V. Yakovlev, V.V. Pai and G.E. Kuz'min \\ Lavrentyev Institute of Hydrodynamics, Siberian Division of Russia Academy of Sciences, \\ Novosibirsk 630090, Russia
}

\begin{abstract}
This paper deals with developing a remote electromagnetic technique for studying shock-wave behavior of porous multi-component materials. The main peculiarity of the method proposed is that both pulse magnetic field source and inductive gauge are placed outside the tested sample. That is why this procedure does introduce no disturbances to observed processes. At the same time the method allows one to measure local parameters of shock loading in porous materials. The technique is illustrated by the application to constructing shock Hugoniots for materials with strongly different physical properties such as alumina, wheat flour and their mixture.
\end{abstract}

Résumé : L'article présente une méthode électromagnétique pour l'étude du comportement sous choc des matériaux composites poreux. La particularité principale de la méthode proposée réside dans le fait que la source du champ magnétique pulsé aussi bien que le senseur d'induction sont placés en dehors de l'échantillon. C'est pourquoi aucunẹ perturbation n'est apportée aux processus étudiés. En même temps, la méthode permet de mesurer les paramètres locaux de l'arrivée de l'onde de choc dans les matériaux poreux. Cette méthode est illustrée par l'application à la construction des courbes d'Hugoniot, pour des matériaux à caractéristiques physiques tres différentes, tels que l'alumine et le mélange alumine et farine de froment.

\section{INTRODUCTION}

For an understanding of the processes that occur under explosive compaction of composite porous bodies a reliable information on the character of the stressed state of the medium being loaded should be available. At present there is a number of experimental techniques for determining the shock parameters in powdered and other heterogeneous media. All of them fall into two main types: local and remote-control techniques. Local methods involve various electric contact techniques providing determination of the shock wave velocity; procedures for measuring pressure by manganin or piezoelectric gauges; electromagnetic method by Zavoiskii for measuring the mass velocity of a material behind a shock wave. The distinctive feature of such experimental procedures is that the gauges are inserted in the substance specimen being studied. The effect of a shock wave on the gauges is recorded and based on these records the state of the medium is restored. Such gauges introduce random distortions in material stressed state. Therefore, the complexity of a correct estimate of the accuracy of such methods is, as a rule, comparable with that of the initial task of the study. High-speed photography, flash radiography, and electromagnetic techniques are among the remote-control procedures that do not require embedment of the gauges in the material under test. Though well adopted for studying the processes in transparent media, the high-speed photography is practically unsuitable for determining shock-wave parameters in continuous and porous materials. Using this method one can register only the moment of the shock wave arrival at the outer boundary of a specimen. The flash radiography is convenient for determining the shock configuration, but is of low resolution. which does not allow for the measurement of shock parameters to a good accuracy. The most promising for application in studying shock wave propagation in porous media are different modifications of electromagnetic technique based on the original Zavoiskii method (see [1] for discussion) but without local gauges.

It seems likely that application of a remote-control electromagnetic technique in solving the problems of shock-wave loading of powders was first proposed in [2]. The specimen of a powdered material was placed in a uniform magnetic field. A signal from a measuring coil placed beyond the specimen appeared 
simultaneously with the onset of shock-wave compaction. That enabled the author [2] to draw a conclusion on the applicability of such a scheme for determining the shock parameters in powders. The related works were reported by J.N. Fritz with colleagues in the papers $[3,4]$. The compression of a magnetic flux by a system of shock waves in an ideally compacted material that can transit instantaneously from a nonconducting state to an unlimited-conductivity state was discussed most thoroughly in [5].

In the present paper we consider a remote electromagnetic technique for determining the mass velocity behind a shock wave in powdered, fibrous, and other heterogeneous media. To demonstrate an application of the technique we give shock Hugoniots of $\mathrm{Al}_{2} \mathrm{O}_{3}$ powder with the initial bulk density of $1.06 \mathrm{~g} / \mathrm{cm}^{3}$. wheat flour of $0.65 \mathrm{~g} / \mathrm{cm}^{3}$ and their mixture (1:1 by weight) of $0.87 \mathrm{~g} / \mathrm{cm}^{3}$ over the pressure range 0.8-5 GPa. The shock wave velocity is measured by the standard electric contact technique.

\section{THEORETICAL ANALYSIS}

Let us investigate electromagnetic processes that take place under shock-wave compaction of a flat
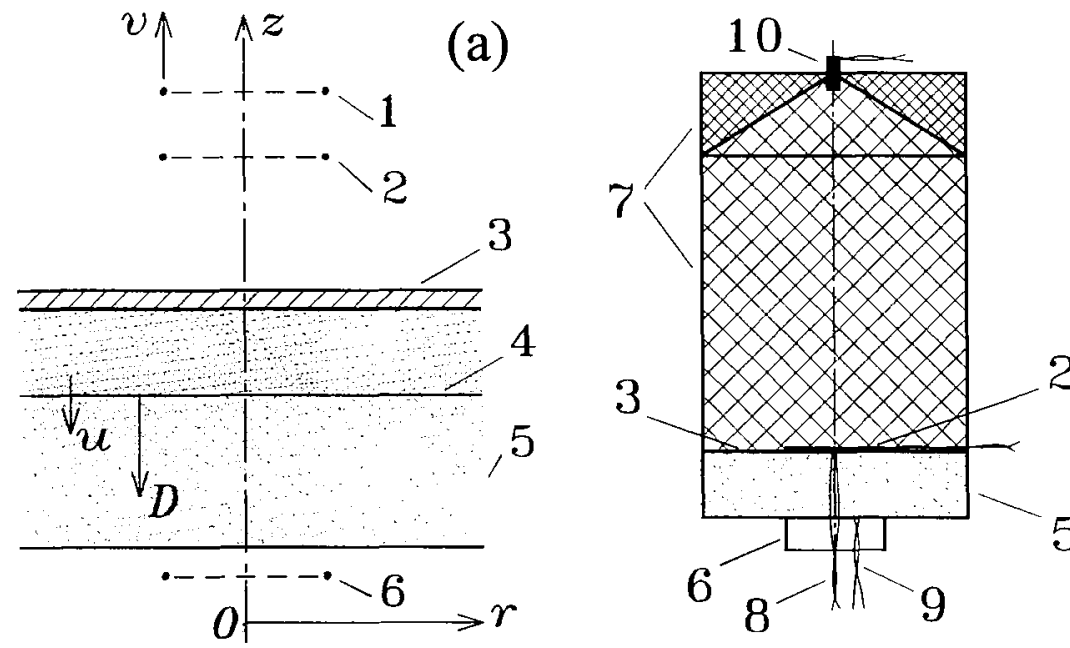

(b)

Figure 1. Shock compression scheme (a) and experimental set-up (b), 1 - radiator image, 2 - radiator, 3 - metallic plate, 4 - shock wave, 5 - powder, 6 - inductive gauge, 7 - explosive charge, 8, 9- contact gauges, 10 - detonator.

unbounded powder layer placed in a magnetic field produced by external sources. The specimen with the measuring elements is shown schematically in Fig. 1. There is a flat metallic plate 3 on the surface of the powder layer 5. A source current coil 2 lying on the plate excites the magnetic field (in Fig. 1(a) the coil is sketched as a section of a single circular turn). Below the powder layer there is an inductive gauge 6 at which the registered electromotive force appears. The gauge is a cylindrical coil shown in Fig. 1(a) as a circular turn. For brevity the current coil 2 exciting the magnetic field is called the radiator; and the coil 6 , from which the signal is taken, the observer. Since the measuring elements (as well as commonly used specimens of substances to be studied) compose an axially symmetrical system, we use the cylindrical coordinates $r, z, \varphi$. The planes of the circular turns under consideration are perpendicular to the plane $r, z ;$ therefore, each turn is shown in Fig. 1(a) as a pair of points.

To analyze the electromagnetic processes in the powder and the measuring system we utilize all electric image approach [6]. Starting from the time $t_{0}$, constant current $I$ flows through the radiator 2. Let 
$\sigma_{0}, \delta_{0}$ be the volume conductivity and the thickness of the plate 3 , respectively. The initial volume conductivity of the powder 5 is equal to zero. Then the magnetic field generated by the radiator below the plate is a superposition of the fields of the standing radiator 2 and its moving image 1 . In this case the direction of the current in the image is opposite to that in the radiator. The image moves away from the radiator with the velocity $v=2 /\left(\mu_{0} \sigma_{0} \delta_{0}\right)$ [6], where $\mu_{0}$ is the magnetic permeability. When $t=t_{0}$ the position of the image coincides with that of the radiator. Let a vector potential due to the radiator 2 placed at the coordinate origin be $\mathbf{A}_{0}(r, z)=\mathbf{e}_{\varphi} A_{0}(r, z)$.

Then the vector potential produced by the radiator-image system under the plate is as follows

$$
\mathbf{A}(r, z, t)=\mathbf{A}_{0}\left(r, z-z_{0}\right)-\mathbf{A}_{0}\left(r, z-z_{0}-v\left(t-t_{0}\right)\right),
$$

where $z_{0}$ is the radiator coordinate. In the observer 6 , which is a singular unclosed turn of radius $R$ and length $L=2 \pi R$ with the coordinate $z=0$, the induction electromotive force will be

$$
U(t)=-\frac{\partial}{\partial t} \mu_{0} L A(r, 0, t)
$$

since $\int_{S} \mathbf{B d S}=\mu_{0} \oint \mathbf{A} d \mathbf{l}$, where $\mathbf{B}$ is the magnetic induction, $d \mathbf{l}$ is an element of the arc turn 6 .

In the other notation

$$
U(t)=-\left.\mu_{0} v L \frac{\partial A_{0}\left(r, z-z_{0}-v\left(t-t_{0}\right)\right)}{\partial z}\right|_{z=0}
$$

Let a switching-off (failure) of the radiator 2 take place at the moment $t_{1}>t_{0}$. Then the field under the plate will be generated by the radiator and the image, which are $v\left(t_{1}-t_{0}\right)$ distant from each other and move at the velocity $v$. In this case the vector potential of the magnetic field when $t \geq t_{1}$ is as follows

$$
\mathbf{A}(r, z, t)=\mathbf{A}_{0}\left(r, z-z_{0}-v\left(t-t_{1}\right)\right)-\mathbf{A}_{0}\left(r, z-z_{0}-v\left(t-t_{0}\right)\right)
$$

Accordingly,

$$
U(t)=-\mu_{0} v L\left(\left.\frac{\partial A_{0}\left(r, z-z_{0}-v\left(t-t_{1}\right)\right)}{\partial z}\right|_{z=0}-\left.\frac{\partial A_{0}\left(r, z-z_{0}-v\left(t-t_{0}\right)\right)}{\partial z}\right|_{z=0}\right) .
$$

Assume that a plane shock wave 4 (Fig. 1(a)) enters the powder through the plate 3 at the moment $t_{2} \geq t_{1}$ with the velocity $D$ and the mass velocity $u$ that equals the plate velocity.

Let the powder retain insulating properties upon shock loading, which holds, for instance, for ceramic materials $\mathrm{Al}_{2} \mathrm{O}_{3}, \mathrm{SiO}_{2}$, etc. Then the velocities of the radiator and the image are $u-v$, and the signal registered by the observer 6 becomes equal to

$$
\begin{aligned}
U(t)= & -\mu_{0}(u-v) L \\
& \times\left(\left.\frac{\partial A_{0}\left(r, z-z_{0}+(u-v)\left(t-t_{2}\right)-v\left(t_{2}-t_{1}\right)\right)}{\partial z}\right|_{z=0}-\left.\frac{\partial A_{0}\left(r, z-z_{0}+(u-v)\left(t-t_{2}\right)-v\left(t_{2}-t_{0}\right)\right)}{\partial z}\right|_{z=0}\right) .
\end{aligned}
$$

Now consider the case when under shock-wave compaction the powder acquires metallic conductivity of the appropriate continuous material when the mass velocity is $u>u_{*}$, where $u_{*}$ is the velocity at which 
the conductivity is "triggered". In strong shock waves realized in dynamic regimes of powder compaction, the width of the shock front, where the mass velocity, density, and, therefore, the material conductivity change significantly, is $\sim(1 \div 2) d$, where $d$ is the particle diameter [7]. Let us estimate the shielding action of the shock front on the magnetic field generated by the radiator 2 . Immediately after the shock wave arrival to the powder the magnetic field of the radiator and the image is "frozen" in the resulting monolithic layer. Let us turn to the reference system that moves with the plate with the velocity $u$. At the initial instant $t=t_{2}$ the magnetic field under the plate 3 is a superposition of the fields of the radiator 2 and the image $1 A_{0}\left(r, z-z_{0}\right)-A_{0}\left(r, z-z_{0}-v\left(t_{2}-t_{1}\right)\right)$. In this reference system the shock wave is a plane material layer moving with the velocity $\sim\left(u_{*}-u\right)$ towards the magnetic field sources. The thickness of the layer is $\sim d$ and the conductivity is $\sim \sigma$ (the conductivity of the monolith). The magnetic Reynolds number $\operatorname{Re}_{m}=\left(u-u_{*}\right) \delta / v_{m}$, where $v_{m}=1 /\left(\mu_{0} \sigma\right)$ characterizes the disturbance of the magnetic field by the shock front [8]. Since the undisturbed field is independent of time, provided that $\operatorname{Re}_{m} \ll 1$ one can consider the magnetic field coincident with that produced by the external sources in the nonconducting medium. With characteristic values of $u-u_{*} \approx 10^{3} \mathrm{~m} / \mathrm{s}$ in strong shock waves and $\sigma \approx 0.6 \cdot 10^{8} \mathrm{Ohm} \cdot \mathrm{m}$ (copper), this condition is satisfied for the powders with particles of less than $3 \mu \mathrm{m}$ in size. For coppercontaining powders and aluminum alloy powders this approximation is valid for the particles whose size is greater by an order of magnitude. If the condition is met, the voltage registered by the observer 6 is described by the expression

$$
U(t)=\mu_{0} u L\left(\left.\frac{\partial A_{0}\left(r, z-z_{0}+u\left(t-t_{2}\right)-v\left(t_{2}-t_{1}\right)\right)}{\partial z}\right|_{z=0}-\left.\frac{\partial A_{0}\left(r, z-z_{0}+u\left(t-t_{2}\right)-v\left(t_{2}-t_{0}\right)\right)}{\partial z}\right|_{z=0}\right) .
$$

Given the distributions $A_{0}(r, z)$, the relations obtained make it possible to determine the velocity

Thus, the analysis has shown that the mass velocity bebind the shock wave can be determined from the electromotive force excited in the inductive gauge. It can be calculated both for porous materials retaining the insulating properties behind the wave and for materials acquiring the monolithic conductivity. To determine the mass velocity using these formulas one should calculate the vector potential under shock loading conditions.

Let us describe the methods for calculating the vector potential produced by the radiator. As follows from [8], the vector potential of the linear circular current has only one component of the potential - along the $\varphi$-axis:

$$
A_{\varphi}=A_{\varphi}(r, z)=\frac{I}{2 \pi} \int_{0}^{\pi} \frac{a \cos \varphi d \varphi}{\sqrt{a^{2}+r^{2}+z^{2}-2 a r \cos \varphi}}
$$

where $a$ is the radius of a turn. Using the parameter $k$ such that $k^{2}=4 a r /\left((a+r)^{2}+z^{2}\right)$ we write the final expression for the magnetic potential $A_{\varphi}$ :

$$
A_{\varphi}=\frac{I}{\pi k}(a / r)^{1 / 2}\left[\left(1-k^{2} / 2\right) K(k)-E(k)\right]
$$

Here $K, E$ are the complete elliptic integrals for the first and second kind.

In view of the linearity of the electrodynamics equations it is obvious that the vector potential and its derivatives for multiple-turn coils are calculated as a sum of the potentials of individual turns.

It should be recognized that the required mass velocity is not expressed from the obtained equations in an explicit form except for the initial instance. Because of this, generally the calculations of the mass velocity from the formulas obtained have conducted with the iteration method on the computer. At the initial time the mass velocity is proportional to the inductive gauge signal. 


\section{EXPERIMENTS AND DISCUSSION}

On the grounds of the theoretical analysis performed and the results of computer calculations an experimental scheme has been developed which realizes the technique for studying shock waves in porous and composite materials. The sample of the porous material being investigated is placed in the magnetic field that is created by the pulsed source of current. Owing to shock loading the magnetic field is changing and its changes are indicated by the inductive gauge that is located outside the sample. The induced electric signal in the gauge involves information about the structure and parameters of the shock wave in the loaded sample.

The experimental set-up is shown in Fig. 1(b). The radiator 2 is a 15-turn spiral coil $40 \mathrm{~mm}$ in diameter made of copper wire of rectangular section. Each turn is $1.0 \mathrm{~mm}$ wide and $30 \mu \mathrm{m}$ thick, so the coil is practically flat. The observer 6 is a coil $40 \mathrm{~mm}$ in diameter made as a 10-turn solenoid of $0.3-\mathrm{mm}$ copper wire. The strength of current flowing through the radiator is $5.7 \mathrm{~A}$. The powder specimens are of $5 \mathrm{~mm}$ in height with the aluminum cover plate of thickness $0.3 \mathrm{~mm}$. The geometric parameters of the experimental set-up, that is, the dimensions and positions of the specific elements, are selected on the base of the preliminary computations to attain the best accuracy and sensitivity of the method. The velocity of a plane shock wave excited in the specimens by a composite explosive charge 7 is measured in the ordinary way using the electric contact gauges 8 and 9 . A representative oscillogram of the inductive gauge signal is shown in Fig. 2. The data are given in Fig. 3 as the pressure-mass velocity-plots for three powders under study: alumina with the initial density of $1.06 \mathrm{~g} / \mathrm{cm}^{3}$, wheat flour of $0.65 \mathrm{~g} / \mathrm{cm}^{3}$ and their mixture (1:1 by weight) of $0.87 \mathrm{~g} / \mathrm{cm}^{3}$. The mean powder particle size is approximately equal to $50 \mu \mathrm{m}$.

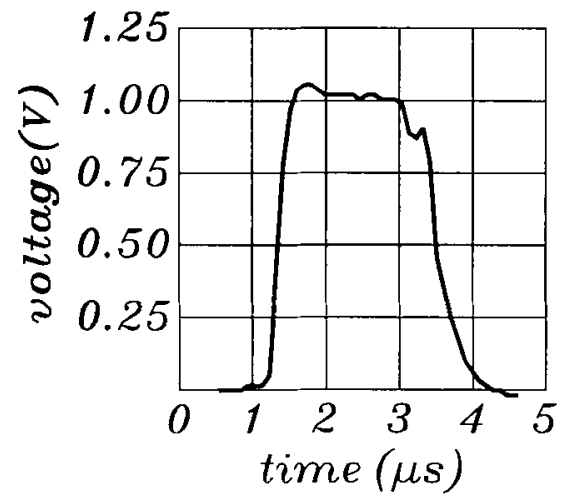

Figure. 2. Typical form of the inductive gauge signal.

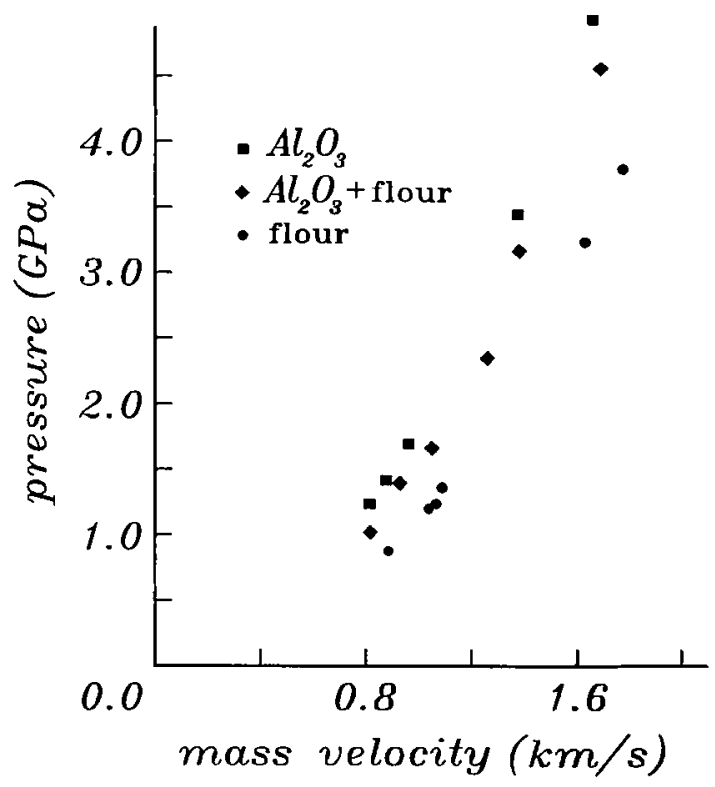

Figure 3. Shock-wave results for three powders.

As is evident from Fig. 2 the sensitivity of the method is indeed very good. The high accuracy of the technique is due to the experimental determination of magnetic induction and its derivatives in a constant magnetic field are not required. It is of essential advantages of this method as compared to the original technique of Zavoiskii [1] and to the methods reported in [3, 4]. Note, in addition, that these works did not deal with the investigation of the shock-wave processes in porous multi-component materials. Moreover, the original Zavoiskii method does not belong to the category of undisturbing techmiques. 


\section{CONCLUSION}

The remote electromagnetic technique is developed for measuring the mass velocity behind the shock waves. The procedure is applicable for studying the processes of dynamic loading of multi-component powders and other composite materials. The method is based on the thorough analysis of the physical model of the electromagnetic processes in porous materials under shock loading. This technique is of high precision and sensitivity. The measurement procedure does introduce no disturbances to observed processes. At the same time the method makes it possible to determine local parameters of shock loading in porous materials. The shock $(p, u)$-plots for $\mathrm{Al}_{2} \mathrm{O}_{3}$ powder, wheat flour and their mixture $(1: 1 \mathrm{by}$ weight) of bulk density are constructed.

\section{Acknowledgments}

The research was partially supported by the Russian Foundation for Fundamental Research (project 94-01. 00999a) and the Los Alamos National Laboratory (contract No. 4540Q0015-35).

\section{References}

[1] Zaitsev V.M., Pokhil P.F., Shvedov K.K., Dokl. Acad. Nauk USSR 132 No. 6 (1969) 1339-1340.

[2] Nesterenko V.F., "Contactless technique for measuring the parameters of shock-compacted metals". 3rd All-Union Symposium on Pulsed Pressures, Moscow 16-18 October 1979, S.S. Batsanov Cd. (Acad. Sci. USSR, Moscow, 1979) pp. 14-15.

[3] Hayes B., Fritz J.N., "Measurement of mass motion in detonation products by an axially-symmetric electromagnetic technique", Fifth Symp. (International) on Detonation, Pasadena 18-21 August 1970. S.J. Jacobs and R. Roberts Eds. (US Government Printing Office, Washington, 1972) pp. 447-454.

[4] Fritz J.N., Morgan J.A., Rev. Sci. Instrum. 44 No. 2 (1973) 215-221.

[5] Bichenkov E.I., Gilev S.D., Ryabchun A.M., Trubachev A.M., Prikl. Mekh. Tekh. Fiz. No. 3 (1987) 15-24.

[6] Maxwell J.C., Treatise on electricity and magnetism (Nauka, Moscow, 1989, V.2) pp. 237-238

[7] Nesterenko V.F., Impulse loading of heterogeneous materials (Nauka, Novosibirsk, 1992) pp. 24-26.

[8] Landau L.D., Lifshits E.M., Electrodynamics of continua (Nauka, Moscow, 1982) pp. 164, 319. 